\title{
General health literacy assessment of Iranian women in Mashhad
}

\author{
Lida Jarahi ${ }^{1}$, Reza Asadi ${ }^{2}$, Hamid Reza Hakimi ${ }^{3}$
}

${ }^{1}$ Community Medicine Specialist, Associate Professor, Department of Community Medicine, Faculty of Medicine, Mashhad University of Medical Sciences, Mashhad, Iran

${ }^{2} \mathrm{MD}, \mathrm{PhD}$, Education Development Center, Mashhad University of Medical Sciences, Mashhad, Iran

${ }^{3}$ MD, Faculty of Medicine, Mashhad University of Medical Sciences, Mashhad, Iran

\section{Type of article: Original}

\begin{abstract}
Background: In women's health, literacy determines their participation in self and family health promotion. Low health literacy is as barrier for understanding medical recommendations, disease prevention and health care.

Aim: To assess women's health literacy and relative factors in Mashhad (Iran).

Methods: Women referring to healthcare centers in Mashhad in 2012 and 2013, participated in this crosssectional study by stratified sampling method. The validated Persian version of Rapid Estimate of Adult Literacy in Medicine-revised questionnaire was used. Vocabulary comprehension and reading scores of health literacy was assessed. Comparisons were done in demographic subgroups by ANOVA, Mann-Whitney U, Kruskal-Wallis, Pearson correlation coefficient, and Chi-Square tests.

Results: In total, 250 women with a mean age of $32.1 \pm 10.23$ years and the mean education level of $10.58 \pm 3.67$ years were studied. The mean reading score was $11.58 \pm 2.51$ and the mean vocabulary comprehension score was 17.24 \pm 4.73. Participants' health literacy score had positive correlation with age and education, and significant difference in health literacy scores between occupational groups was seen. Housewives' health literacy scores were lower than others $(\mathrm{p}<0.05)$.

Conclusion: Low health literacy was a common problem amongst younger women, especially among women who had less education. These women are at risk of early marriage and child bearing and require more health care. Health care professionals should use effective methods for easier transfer recommendation, also, producing medical information booklets, texts, and videos for different community subgroups through public media or even in cyberspace with clear and common words consisting of essential information.
\end{abstract}

Keywords: Health Literacy, Health Promotion, Women, Iran

\section{Introduction}

Different understanding about health issues between physicians and patients are of great importance, since they may reduce the impact of health education and health promotion measures (1). Health literacy is the ability to read, understand, and apply printed health-related information, follow doctors' prescriptions and use the obtained information for proper use of healthcare services $(1,2)$. High health literacy empowers individuals to understand, apply health-related information and instructions and find ways to use healthcare services in order to meet their needs; including familiarity with modes of transmission and prevention of common diseases and treatment methods, following doctors' suggestions, ability to understand health brochures, and use health insurance (1-3). In a community with low health literacy, understanding and applying health-related information and guidelines, following instructions for diagnostic screening, treatment adherence is poor $(3,5)$. Unfamiliarity of the medical community with concept of health literacy, encumbers the doctor-patient relationship and might lead to inaccurate prediction of treatment outcomes (especially in chronic non-communicable diseases, which require patients' understanding of their conditions and their cooperation in the course of treatment $(2,3)$. Ignorance of healthcare

\section{Corresponding author:}

Dr. Reza Asadi, Addiction Research Center, Mashhad University of Medical Sciences, Mashhad, Iran.

Tel: +9891531108683, Fax: +9851138829263, Email: AsadiR1@mums.ac.ir

Received: May 14, 2016, Accepted: June 21, 2017, Published: November 2017

iThenticate screening: June 09, 2017, English editing: July 28, 2017, Quality control: August 02, 2017

(C) 2017 The Authors. This is an open access article under the terms of the Creative Commons Attribution-NonCommercialNoDerivs License, which permits use and distribution in any medium, provided the original work is properly cited, the use is non-commercial and no modifications or adaptations are made. 
system planners regarding health literacy levels can lead to inaccurate predictions of the achieved results, and low health literacy is linked with adverse health outcomes and inappropriate use of healthcare services (2-4). Inadequate health literacy is associated with poor self-reported health status, poorly managed chronic diseases, poor physical and mental health, less frequently used emergency departments and hospital services, and has negative effects on women's health, ability of selfcare child care, their families' health, directions for taking medication and so on (5-8). Some research reported that there were frequent mismatches in concepts of health-related material and peoples' perceptions of reading medical information, which points to difficulty of text readability or unfamiliar words or scientific names (9). So, assessing peoples' literacy levels leads to the question of how material is difficult or unclear for the general population $(3,9)$. In regard to the growing importance of health literacy in the improvement of community health, especially in a developing country, the aim of this study was to assess the health literacy level of Iranian women referring to healthcare centers in Mashhad.

\section{Material and Methods}

\subsection{Research design and setting}

This cross-sectional study was conducted on women attending healthcare centers of Mashhad University of Medical Sciences, Iran in 2012 and 2013. The inclusion criteria were residing in Mashhad, reading and writing abilities, and consent to participate in the study. . Foreign residents, illiteracy, having a mental disease, and aged younger than 18 years were the exclusion criteria. People who usually wore glasses for reading, were asked to wear them for the test.

\subsection{Sampling}

Stratified sampling was applied and simple sampling was employed in each center. Sample size was determined based on a pilot study on 20 persons and with sample size formula for estimating a single mean with specified precision, with $\mathrm{Z}_{\alpha}=1.96, \mathrm{SD}=0.05, \mathrm{~d}=0,65$ and design effect $=2$.

\subsection{Measurement tool}

The health literacy measurement tools of Rapid Estimate of Adult Literacy in Medicine (REALM-R), is an eightitem word recognition instrument, designed for rapidly screening people for potential health literacy problems $(6,7$, 10). This study questionnaire was designed based on REALM-R questionnaire, which was normalized according to Persian language and culture. We assessed vocabulary comprehension in addition to read scores in a modified Persian version. The total vocabulary comprehension score, which was considered as an indicator of health literacy, was divided into three categories. Those with scores which were lower than or equal to $50 \%$ of the total score (12 out of 24 points) were considered to have inadequate or low health literacy, and those with scores which were between $50 \%$ to $75 \%$ of the total score (18 out of 24 points) were considered to have marginal health literacy. Also, those with scores more than $75 \%$ of the total score were considered to have adequate health literacy. Validity of questionnaire was assessed by expert primary care physicians, and reliability was determined by Cronbach's alpha. The first section of the questionnaire was about socio-demographic variables, and subsequently, participants completed the health literacy questionnaire. In the Persian questionnaire, twelve items were read by each participant for assessing reading scores, and after that, participant expressed their own comprehension of each medical word. Each participant was given a list of the words. They were asked to point at the words and read each of them aloud. If the person took more than five seconds on a word, they were encouraged to move on to the next word. Medical words included "anxiety, osteoporosis, anemia pap smear, breast cancer, mammography, menopause, domestic violence, cesarean section, ovarian cyst, spotting and female reproductive infection. Two easy-to-read medical words at the beginning of the questionnaire were not scored and were positioned to decrease test anxiety and enhance confidence. Trained interviewers asked participants to read each word and then explain its meaning. The interviewer noted participants' definitions. For example; for common cold, the participant would express the meaning of common cold by saying "In my opinion, the meaning of common cold is..." Finally, each person was given a score from zero to 12 in the reading section (based on the number of words they were able to read correctly) and zero-24 in the vocabulary comprehension (health literacy score) section (knows, rather knows, does not know).

\subsection{Data collection}

After defining the sample clusters based on the number and list of Mashhad city health centers, in each selected center, women referring to healthcare centers to receive health services were invited to participate in the study up to completion of the sample size of each cluster. 
http://www.ephysician.ir

\subsection{Ethical consideration}

The protocol of this study was approved by the Ethics Committee of the Mashhad University Medical Sciences. Our research conformed to legislations on Ethical Principles for Medical Research Involving Human Subjects. Informed consent was obtained from participants before participation.

\subsection{Statistical analyses}

After collecting data through questionnaires, data were analyzed by SPSS version 11.5 (SPSS Inc., Chicago, Illinois, USA). Descriptive statistics including frequency, mean, and standard deviation were used to describe the data, and relationship between variables was examined by ANOVA, Mann-Whitney, Kruskal-Wallis, Pearson correlation coefficient, and Chi-Square tests.

\section{Results}

\subsection{Baseline characteristics}

A total of 250 women participated in the study. The mean age of the participants was $32.18 \pm 10.23$ years, minimum age of participants was 18 and maximum age was 67 years. The mean marital age was $19.2 \pm 3.65$ years. The participants had an average of 1.88 children. The mean education level of participants was $10.58 \pm 4.2$ years. Table 1 shows demographic characteristics of participants. Overall, 155 women (62\%) had visited a doctor during the last 3 months, and among them, 40 (25\%) subjects had referred to a physician for a check-up, 93 subjects (60\%) due to an illness or health problem, 9 patients $(5 \%)$ for both check-up and health problems, and 13 participants $(8 \%)$ due to other causes. In addition, 64 (25\%) participants had a previous history of hospitalization over the last year.

Table 1. Demographic characteristics of the study population

\begin{tabular}{|c|c|c|}
\hline \multicolumn{2}{|l|}{ Variable } & $\mathrm{n}(\%)$ \\
\hline \multirow[t]{4}{*}{ Age group (years) } & $<20$ & $13(5.2)$ \\
\hline & $20-30$ & $104(41.6)$ \\
\hline & $30-40$ & $77(30.8)$ \\
\hline & $>40$ & $56(22.4)$ \\
\hline \multirow[t]{3}{*}{ Education level } & Less than diploma & $109(43.6)$ \\
\hline & High-school diploma & $87(34.8)$ \\
\hline & College education & $54(21.6)$ \\
\hline \multirow[t]{6}{*}{ Job } & Housewives & $206(82.4)$ \\
\hline & Employees & $16(6.4)$ \\
\hline & Student & $16(6.4)$ \\
\hline & Retired & $4(1.6)$ \\
\hline & Workers & $2(0.8)$ \\
\hline & self-employed & $4(2.4)$ \\
\hline \multirow{6}{*}{$\begin{array}{l}\text { Participants' spouses' } \\
\text { job }\end{array}$} & Employee & $41(16.9)$ \\
\hline & Self-employed & $134(55.7)$ \\
\hline & Retired & $12(4.8)$ \\
\hline & Student & $7(2.8)$ \\
\hline & Worker & $41(16.9)$ \\
\hline & Unemployed & $5(1.9)$ \\
\hline \multirow[t]{4}{*}{ Marital status } & Married & $241(96.4)$ \\
\hline & Single & $4(1.6)$ \\
\hline & Divorced & $3(1.2)$ \\
\hline & Widowed & $2(0.8)$ \\
\hline \multicolumn{2}{|c|}{ Pregnancy } & $25(10.0)$ \\
\hline \multicolumn{2}{|c|}{ History of hospitalization } & $64(25.6)$ \\
\hline \multicolumn{2}{|c|}{ Outpatient recent visit to doctor } & $155(62.0)$ \\
\hline
\end{tabular}

\subsection{REALM-R questionnaire results}

Mean of reading score (scored from 0 to 12 ) was $11.58(\mathrm{SD}=0.96)$, the lowest and highest scores were 6 and 12 , respectively. Mean of health literacy score (scored from 0 to 24) was $17.24(\mathrm{SD}=4.55)$, the lowest score was 4 and the highest score was 24. Most of the participants $(n=128,51.2 \%)$ had an adequate level of health literacy; followed by marginal levels in $36.8 \%(n=91)$ and inadequate (low) levels in $12.0 \%$ of participants $(n=30)$. 


\subsubsection{Age}

There was a significant negative correlation between the reading score and age $(r=-0.30, p=0.001)$, also, there was significant positive correlation between the health literacy score and age $(r=0.28, \mathrm{p}=0.006)$. Assessment of the health literacy score in different age groups showed that respectively, the group under 20 years old had lower health literacy, 20 to 30 years old and 30 to 40 years old were after those, and this difference was significant comparing the groups $(\mathrm{p}=0.002,0.004,0.02$ respectively), that means younger women had lower health literacy scores.

\subsubsection{Education}

Also, there was seen a significant positive correlation between women's education years with reading score ( $\mathrm{r}=0.46$, $\mathrm{p}=0.01)$, and health literacy score $(\mathrm{r}=0.38, \mathrm{p}=0.03)$. The Kruskal-Wallis test showed significant differences between level of education and health literacy, that health literacy score in women with less than diploma education was significantly lower than from high school graduates and those who had college education $(\mathrm{p}=0.001)$, also, those with high-school diploma were significantly lower in health literacy score compared to those who had college education $(\mathrm{p}=0.02)$. According to Scheffe's analysis, those with adequate health literacy were 7.4 years older than those with inadequate health literacy $(\mathrm{p}=0.001)$, and were 4.6 years older than those with marginal levels of health literacy $(\mathrm{p}=0.02)$. The Dunnett T3 post-hoc test showed that the marital age of those with adequate health literacy was 2.6 years $(\mathrm{p}=0.002)$ more than those with low levels of health literacy; also, those with marginal health literacy levels were married 1.61 years $(\mathrm{p}=0.04)$ later than those with inadequate health literacy.

\subsubsection{Women's Job}

The ANOVA test showed no significant difference between occupational groups in terms of reading scores $(\mathrm{p}=0.28)$; however, a significant difference was seen in health literacy scores between occupational groups $(\mathrm{p}=0.001)$. After performing post-hoc analysis (Dunnett T3), it was found that housewives' health literacy scores were lower ( 4.3 scores) than the employees and retired women ( $\mathrm{p}=0.02,0.04$ respectively).

\subsubsection{Spouses' Job}

Women, whose spouses were employees, had significantly higher health literacy scores (4.53 scores), compared to those who were workers $(\mathrm{p}=0.001)$; they were also greater $(2.5$ scores $)$ than those with self-employed husbands $(\mathrm{p}=0.01)$.

\subsubsection{Marital Status}

There was a significant positive correlation between age of marriage with reading scores $(\mathrm{r}=0.13, \mathrm{p}=0.04)$, and also health literacy scores $(\mathrm{r}=0.24, \mathrm{p}=0.002)$. The ANOVA test did not show significant differences between health literacy scores and marital status, and not in pregnancy ( $\mathrm{p}=0.45,0.32$ respectively).

\subsubsection{Hospitalization and physician referral}

Women with history of hospitalization had higher health literacy $(\mathrm{p}=0.4)$ but did not show a significant difference between health literacy levels and recent physician referral $(\mathrm{p}=0.14)$.

\section{Discussion}

This study is amongst the first studies to assess the health literacy of women in Iran. The results of this study showed that nearly half of the women who participated in this study, had a literacy level below the adequate level of literacy. Of the participants, $12 \%$ had inadequate, and $36.8 \%$ had marginal health literacy levels. The results of the only conducted survey in five provinces of Iran yielded lower health literacy levels (25.9\%) in participants (5); the difference is probably due to the fact that the current study was only performed on subjects referring to healthcare centers. People with low health literacy, are less informed about their health status and receive less preventive healthcare services (8). In other words, the possibility exists that women using primary healthcare services can benefit from a higher level of health literacy; in fact, this study was conducted among people with higher health literacy $(8,9)$. The results of the national assessment of literacy $(2006)$ in the United States showed that $36 \%$ of adults have limited health literacy (inadequate or marginal health literacy). In other words, these individuals can only understand part of a short simple text (lower than basic health literacy); in longer text, they can find the information, provided that the text is clear and simple (basic health literacy) (2). Health literacy is considered to be an important element in a woman's ability to engage in health promotion and disease prevention activities for themselves and their children. Without adequate understanding of healthcare-related information, it would be difficult or impossible for a woman to make an informed decision, which would result in optimum health of themselves and their families $(9,11)$. In similar studies conducted on pregnant women with diabetes and mothers in 
postpartum phase, the adequate health literacy level was higher than (56-80\%) that of the current study; this might be due to the higher literacy level of participants in those studies (12-14). In this study, years of education was significantly associated with health literacy level. This relationship has been detected in some other studies (11-14). Although based on the findings of this study, health literacy was positively correlated with the level of education, there was a significant percentage of limited health literacy among the higher educated groups; in fact, $25 \%$ of those with a college degree, and more than $40 \%$ of people with a high school and pre-university diploma had inadequate or marginal health literacy; these findings were consistent with those of other studies $(2,4,14)$. Saunders et al. in a review study, suggested that $66 \%$ of people with limited health literacy had high-school diploma or higher education (8). Maybe academic years are not considered as a valid independent indicator of reading comprehension skills and assessment of health literacy, based on the latest education degree, it is not valid $(15,16)$. Our study results indicated a positive relationship between age and health literacy level, but some studies have reported a negative correlation between age and health literacy level $(15,17)$. Some studies could not find a significant association between age and health literacy among pregnant women (17). Finally, marital status was not significantly associated with the level of health literacy in this study. This might be due to the limited number of individuals, referring to healthcare centers; therefore, further research is required in this area. Assessment and determination of the level of functional literacy are vital for healthcare systems in order to design effective methods for conveying information to people who have limited reading comprehension skills. In fact, through assessment, we can be ensured that people can understand their health status and follow health guidelines (12). Because of work time in care centers, most women who referred to health centers were housewives, and working women had less participation in this study.

\section{Conclusions}

This study revealed that nearly half of the women in this study had inadequate levels of literacy, which is a common problem in healthcare centers of Mashhad. Healthcare center staff and health professionals should use effective methods for information transfer and easier health recommendation, and produce medical information booklets, texts, and/or videos for different subgroups of the community in public media or even in cyberspace with clear and common words consisting of essential information.

\section{Acknowledgments:}

This research was supported by the Research Council of Mashhad University of Medical Sciences, Mashhad, Iran. The result of this paper is based on an MD thesis. We thank our colleagues in Mashhad health centers for greatly assisting the research.

\section{Conflict of Interest:}

There is no conflict of interest to be declared.

\section{Authors' contributions:}

All authors contributed to this project and article equally. All authors read and approved the final manuscript.

\section{References:}

1) Paasche-Orlow MK, Parker RM, Gazmararian JA, Nielsen-Bohlman LT, Rudd RR. The prevalence of Limited health literacy. J Gen Intern Med. 2005; 20(2): 175-84. doi: 10.1111/j.1525-1497.2005.40245.x. PMID: 15836552, PMCID: PMC1490053.

2) Shieh C, Halstead JA. Understanding the impact of health literacy on women's health. Obstet Gynecol Neonatal Nurs. 2009; 38(5): 601-10. doi: 10.1111/j.1552-6909.2009.01059.x. PMID: 19883483.

3) Corrarino JE. Health literacy and women's health: challenges and opportunities. J Midwifery Womens Health. 2013; 58(3): 257-64. doi: 10.1111/jmwh.12018. PMID: 23631442.

4) Haghighi ST, Lamyian M2, Granpaye L. Assessment of the level of health literacy among fertile Iranian women with breast cancer. Electron Physician. 2015; 7(6): 1359-64. doi: 10.14661/1359. PMID: 26516442, PMCID: PMC4623795.

5) Banihashemi SA, Amirkhani MA, Haghdoost AA, Alavian SM, Asgharifard H, Baradaran H, et al. Health literacy and the affecting factors: a study in five provinces of Iran. J Med Educ Dev Cent. 2007; 4(1): 1-9.

6) Gordon MM, Hampson R, Capell HA, Madhok R. Illiteracy in rheumatoid arthritis patients as determined by the Rapid Estimate of Adult Literacy in Medicine (REALM) score. Rheumatology (Oxford). 2002; 41(7): 750-4. doi: 10.1093/rheumatology/41.7.750. PMID: 12096223. 
7) Shea JA, Beers BB, McDonald VJ, Quistberg DA, Ravenell KL, Asch DA. Assessing health literacy in African American and Caucasian adults: disparities in rapid estimate of adult literacy in medicine (REALM) scores. Fam med. 2004; 46(8): 575-81. PMID: 15343419.

8) Pearson A, Saunders M. Health literacy revisited: what do we mean and why does it matter? Health Promot Int. 2009; 24(3): 285-96. doi: 10.1093/heapro/dap014. PMID: 19372101.

9) McCray A. Promoting Health Literacy. J Am Med Inform Assoc. 2005; 12(2): 152-63. doi: 10.1197/jamia.M1687. PMID: 15561782, PMCID: PMC551547.

10) Bass PF, Wilson JF, Griffith CH. A Shortened Instrument for Literacy Screening. J Gen Intern Med. 2003; 18(12): 1036-8. doi: 10.1111/j.1525-1497.2003.10651. PMID: 14687263, PMCID: PMC1494969.

11) Ferguson B. Health literacy and health disparities the rolethey play in maternal and child health. Nurs Womens Health. 2008; 12(4): 288- 98. doi: 10.1111/j.1751-486X.2008.00343.x. PMID: 18715376.

12) Pati S, Feemster KA, Mohamad Z, Fiks A, Grundmeier R, Cnaan A. Maternal Health Literacy and Late Initiation of Immunizations Among an Inner-City Birth Cohort. Matern Child Health J. 2011; 15(3): 38694. doi: 10.1007/s10995-010-0580-0. PMID: 20180003.

13) Pati S, Mohamad Z, Cnaan A, Kavanagh J, Shea JA. Influence of Maternal Health Literacy on Child Participation in Social Welfare Programs: The Philadelphia Experience. Am J Public Health. 2010; (100): 1662-5. doi: 10.2105/AJPH.2009.172742. PMID: 20634468, PMCID: PMC2920956.

14) Endres LK, Sharp LK, Haney E, Dooley SL. Health literacy and pregnancy preparedness in pregestational diabetes. Diabetes Care. 2004; 27(2): 331-4. doi: 10.2337/diacare.27.2.331. PMID: 14747209.

15) Carthery-Goulart MT, Anghinah R, Areza-Fegyveres R, Santoro Bahia V, Dozzi Brucki SM, Damin A, et al. Performance of a Brazilian population on the test of functional health literacy in adults. Rev Saúde Pública. 2009; 43(4): 631-8. doi: 10.1590/S0034-89102009005000031. PMID: 19488667.

16) Safeer RS, Keenan J. Health Literacy: The gap between physicians and patients. Am Fam Physician. 2005; 72(3): 463-8. PMID: 16100861.

17) Jovic-Vranes A, Bjegovic-Mikanovic V, Marinkovic J. Functional health literacy among primary healthcare patients: data from the Belgrade pilot study. J Public Health (Oxf). 2009; 31(4): 490-5. doi: 10.1093/pubmed/fdp049. PMID: 19454605. 